Management problems

\title{
Pitfalls in the interpretation of common biochemical tests
}

\author{
Ruth M Ayling
}

\begin{abstract}
Summary
This review considers some of the more common problems in the interpretation of the results of biochemical tests and, where possible, highlights ways in which errors can be identified or avoided.
\end{abstract}

Keywords: biochemical tests

Department of Clinical Biochemistry, King's College Hospital, Denmark Hill, London SE5 9RS, UK R Ayling

Submitted 7 June 1999 Accepted 13 August 1999
The development of multichannel automated analysers has led to the majority of hospital patients having a biochemistry profile performed at some point during their care. Whilst these investigations are often viewed as simple tests, there are many pitfalls in the interpretation of their results, potential for error putting the patient at risk of over investigation or even erroneous diagnosis. There are many reasons for spurious abnormalities which can falsely raise or lower the concentrations of analytes, both prior to sample handling or during the analytical process itself. Lack of tissue specificity of enzymes may make interpretation of their activities difficult without additional tests. Hitherto unsuspected disease may also contribute to confusion in test interpretation.

\section{Reference ranges}

Biochemical tests are usually interpreted in the light of a quoted reference range or, more correctly, reference interval. The reference range is usually determined as the interval into which the central $95 \%$ of apparently healthy people fall. If the distribution is Gaussian this is the range two standard deviations above and below the mean. With non-Gaussian distributions the range is obtained by deleting $2.5 \%$ at the upper and lower ends of ranked values. In other words, one in 20 (ie, $5 \%$ ) apparently healthy people will have a value outside this range. The finding of a value outside the quoted reference range does not therefore necessarily indicate the presence of a pathological cause and the more tests performed which are independent variables, the greater the probability of finding a result outside the reference range. Similarly, the presence of disease is not excluded by a result within the reference range. Different values obtained by sequential analyses on the same patient could be due to a genuine change in biochemical status or to laboratory imprecision. If the results of two tests performed on the same patient on two occasions under identical conditions differ by more than 2.8 times the analytical standard deviation, a genuine change in analyte concentration is more than $95 \%$ likely to have occurred. ${ }^{1}$ However, biological factors can cause intra-individual variation, influencing the plasma concentrations of some analytes. For example, diet can affect plasma triglyceride concentration and urinary calcium excretion, circadian variation is well recognised for cortisol and plasma iron, and posture can increase the concentration of many analytes, including albumin, calcium, cholesterol, triglycerides and amylase. In women, cholesterol, total protein, albumin and fibrinogen alter during the menstrual cycle and seasonal variation is recognised for vitamin D and growth hormone. Pyrexia, drugs and the acute phase response may lead to variation during acute illness. $^{2}$

\section{Sodium}

Mild hyper- and hyponatraemia are common in the hospital population. Spurious abnormalities of sodium can be due to sampling error, for example, venepunctures from above infusions of sodium bicarbonate or other solutions. Other clues that the sample has been contaminated by intravenous fluid will be a decrease in its total protein and albumin concentration with respect to previous samples and a high glucose concentration if the infusion fluid contains dextrose. After collection, evaporation of water from the sample may produce hypernatraemia. With certain methods of analysis, for example, flame emission spectrophotometry and use of an indirect ion-selective electrode, where dilution of the specimen in a large volume of solution is required, pseudohyponatraemia may occur in patients with severe triglyceridaemia or paraproteinaemia and very rarely may arise secondary to severe hypercholesterolaemia. ${ }^{3}$ This is because part of the plasma water has been replaced with the lipid or protein; the sodium concentration within the plasma water is normal but there is less water within each 
litre of plasma. Plasma osmolality (concentration/kg solvent) will be normal in this situation.

\section{Potassium}

Whilst the majority of total body potassium is intracellular, it is plasma potassium that is usually measured. Spurious hypo- and hyperkalaemia are common and arise from movement of potassium between intra- and extracellular compartments in vitro The most common cause of pseudohyperkalaemia is haemolysis, which may not always be visible from inspection. In samples of whole blood which are delayed in transit to the laboratory, particularly if stored at $4^{\circ} \mathrm{C}$, there will be loss of potassium from red blood cells into the plasma even when there is no evidence of haemolysis. Clues to this from other biochemical tests might include a co-existing increase in phosphate. A predisposition to pseudohyperkalaemia alone may be familial. ${ }^{4}$ In patients with abnormalities causing increased white cell counts, such as prolymphocytic leukaemia ${ }^{5}$ or increased platelet counts, for example in myeloproliferative disorders ${ }^{6}$ and Kawasaki's disease, ${ }^{7}$ cellular lysis and release of potassium is known to cause pseudohyperkalaemia, especially when serum samples are used. To avoid this, such patients' blood should be collected into a tube containing heparin and taken straight to the laboratory. It has been estimated that for every $100 \times 10^{9} / 1$ increase in platelet count, the potassium concentration can rise by up to $0.07-$ $0.15 \mathrm{mmol} / 1 .{ }^{8}$ Even in samples with a normal platelet count the reference range for potassium is higher in serum than plasma due to release from platelets during clotting. Less commonly samples of blood with very high white cell counts may lead to pseudohypokalaemia as the cells take up potassium from the plasma. ${ }^{9}$ A further example of pseudohypokalaemia resulting from potassium uptake by blood cells is that by erythrocytes when blood is taken from a patient just after receipt of intravenous insulin.

\section{Urea and creatinine}

Urea and creatinine are both used as indicators of renal function; creatinine is the more reliable measurement although not ideal. Due to the inverse relationship between glomerular filtration rate (GFR) and plasma creatinine, halving the GFR will approximately double the plasma creatinine. However, for a given individual, a reduction in GFR of this magnitude may result in a plasma creatinine concentration that remains within the reference range. Creatinine arises from creatine phosphate in muscle. The reference range is related to muscle mass and care must be taken not to overlook significant changes in renal function in those patients with small muscle mass which may be indicative of renal failure, although the actual creatinine concentration remains within the quoted reference range. The ingestion of a meat meal can increase plasma creatinine by up to $52 \%$ within $3.5 \mathrm{~h}^{10}$ and measurements should ideally be made fasting.

Urea is synthesised in the liver and is the body's main vehicle of nitrogen excretion. Urea production may be affected by dietary protein content and by absorption of amino acids and peptides from the gut after gastrointestinal haemorrhage. In severe liver disease urea synthesis and hence plasma urea concentration may be decreased, even in renal failure. When the rate of flow through the tubular lumen is low, for example in dehydration, urea is reabsorbed and the plasma concentration rises although creatinine is initially normal.

\section{Calcium}

In the plasma, calcium exists in two major forms, protein-bound (about 46\%) and ionised (about $47 \%$ ), only the latter being physiologically active. Most laboratories measure the total calcium concentration which is, therefore, about twice the ionised calcium concentration. The quoted reference range for total calcium assumes a plasma albumin of $40 \mathrm{~g} / \mathrm{l}$. If this is not so, a correction must be made or otherwise hypercalcaemia may be erroneously diagnosed in association with an albumin concentration greater than $40 \mathrm{~g} / \mathrm{l}$ and hypocalcaemia with one below $40 \mathrm{~g} / \mathrm{l}$. A suitable formula for this correction is:

Corrected plasma calcium $(\mathrm{mmol} / \mathrm{l})=$ measured plasma calcium $+0.02[40-$ albumin concentration $(\mathrm{g} / \mathrm{l})]$

This correction is not valid in the presence of a marked abnormality of hydrogen ion homeostasis or gross dysproteinaemia.

Venous stasis increases total calcium concentration. ${ }^{11}$ The ionised calcium concentration remains unchanged in conditions of venous stasis unless the blood hydrogen ion concentration is altered. Venepuncture using a tourniquet results in haemoconcentration and an increase in the protein concentration of the sam- 


\section{Summary points \\ - a result outside the reference range is not necessarily abnormal \\ - pseudohyperkalaemia may occur in samples with high white cell and platelet counts and blood samples for biochemistry tests on such patients should be taken into tubes containing heparin (green vacutainer) \\ - creatinine is a more reliable indicator of renal function than urea \\ - total calcium concentration should be corrected for the plasma albumin \\ - for accurate determination of glucose concentration specimens should be collected into tubes containing an inhibitor of glycolysis, eg, sodium fluoride \\ - a CK-MB fraction $>6 \%$ can occur after severe exercise, surgery and muscle damage}

Box 1 ple and the total calcium therefore increases. Ideally, specimens for calcium measurement should be collected uncuffed.

EDTA, commonly used as the anti-coagulant in containers for samples for haematology tests, chelates calcium and other divalent metal ions and, if present in a sample, even in minute amounts as a contaminant, can cause spurious hypocalcaemia. As alkaline phosphatase depends on divalent metal ions for activation, its activity in the plasma will also be lowered by EDTA. The usual form of the anticoagulant is potassium EDTA, so a further clue to its presence will be an artefactually raised potassium.

\section{Phosphate}

Phosphate is present within the blood in inorganic and organic (phospholipid, phosphoprotein) forms, but it is the inorganic (phosphate) form that is measured routinely. There are age-related reference ranges for plasma phosphate concentrations. Values are highest in infancy and adolescence when growth is maximal, but throughout childhood the reference range is higher than in adult life. The plasma phosphate concentration is also raised during lactation. Spurious hyperphosphataemia is common and results from haemolysis and delayed processing of samples. With some methods of analysis, spurious hyperphosphataemia has been reported in samples containing high protein concentrations. ${ }^{12}$

\section{Glucose}

The measurement and interpretation of glucose concentrations is an area of much confusion. Plasma glucose is about $12 \%$ greater than that of whole blood because plasma has a higher water content as it contains no red blood cells. In the fasting state there is little difference between arterial, capillary and venous glucose concentrations but after carbohydrate intake glucose concentration in arterial and capillary samples can exceed those of venous samples by as much as $1.8 \mathrm{mmol} / 1 .^{13}$ This is of particular practical importance in the interpretation of oral glucose tolerance tests.

For the measurement of glucose a specimen containing sodium fluoride to inhibit glycolysis and stabilise the glucose concentration is preferred. If blood is collected into a tube without preservative, glucose concentration can decrease by as much as $7 \%$ each hour as a result of glycolysis. ${ }^{14}$ Clinically significant decreases in glucose concentration have been reported even with use of preservative. ${ }^{15}$ If a tube without preservative is used and delay in transit to the laboratory is anticipated, storage at $4^{\circ} \mathrm{C}$ is preferable.

\section{Bilirubin}

Both conjugated and unconjugated bilirubin are broken down by light in a temperature-dependent way. Therefore, specimens for determination of bilirubin in plasma or urine should be wrapped in foil or dark paper and stored in the fridge if analysis is to be delayed.

\section{Liver function tests}

Most hospital laboratory laboratories measure a combination of enzymes, most commonly alkaline phosphatase (AP), aspartate transaminase (AST, previously called glutamate oxaloacetate transaminase), alanine aminotransferase (ALT, previously called glutamate pyruvate transaminase) and gamma glutamyltransferase (GGT). They are often referred to as 'liver function tests (LFTs)', as they are usually being used to find out if there is evidence of liver damage.

$\mathrm{AP}$ belongs to a group of enzymes which hydrolyses phosphate esters. Increases in activities of these enzymes are not specific for liver disease as plasma $\mathrm{AP}$ also arises from bone, intestine, and, during pregnancy, from the placenta. In infants and children the reference range is higher due to an increased bone contribution secondary to rapid growth. Separation of AP isoenzymes can differentiate the tissue of origin.

AST and ALT are enzymes which catalyse the transfer of an $\alpha$-amino group from an $\alpha$-amino to an $\alpha$-oxo acid within cells. AST and ALT are found in many extra-hepatic tissues including heart, skeletal muscle, erythrocytes, lung, brain and kidney, although the extrahepatic contribution in plasma is less for ALT making it more liver specific. Muscle damage, acute cardiac failure and shock may rarely cause an increase in AST as high as 10 times the upper limit of the reference range. Elevation to six times normal has been described in hypothyroidism, ${ }^{16}$ but this is of muscular rather than liver origin and is accompanied by a rise in creatine kinase activity. 


\begin{tabular}{|l|}
\hline Questions \\
\hline Are the following statements true or false? \\
Answers are given on p 169 \\
1 A reference range is derived from the \\
mean and two standard errors above \\
and below it. \\
2 Plasma osmolality will be normal in \\
spurious hyponatraemia secondary to \\
dilution of sample by intravenous \\
infusion fluid and in \\
pseudohyponatraemia in patients with \\
severe triglyceridaemia. 3 \\
3 A patient with a markedly abnormal \\
GFR may have a normal plasma \\
creatinine concentration. \\
4 Plasma glucose is greater than whole \\
blood glucose. \\
5 AST activity may be increased in \\
hypothyroidism.
\end{tabular}

Box 2

GGT is a microsomal enzyme which transfers $\gamma$-glutamyl groups from $\gamma$-glutamyl peptides to amino acids and other peptides. It is often measured as a surreptitious way of screening for alcohol abuse. However, amongst alcoholics without liver disease only half show a raised GGT, the extent of which is related to neither the amount nor the duration of ethanol consumption. A large number of drugs, not just anticonvulsants, increase GGT activity, and rarely activity can be increased in carcinoma of the prostate ${ }^{17}$ and hyperthyroidism. ${ }^{18}$

\section{Creatine kinase}

Creatine kinase (CK) exists as a dimer of $\mathrm{M}$ and $\mathrm{B}$ subunits which combine to form one of three dimers, MM, MB or BB. Skeletal muscle contains mostly $\mathrm{MM}$, cardiac muscle contains about $40 \% \mathrm{MB}$ and brain contains only BB. CK-MM, and hence total CK, activity may be increased in patients with a large muscle mass and shows variation with ethnic origin, being higher in Afro-Caribbeans. ${ }^{19} \mathrm{CK}$ is commonly used as an indicator of myocardial infarction (MI), however its level in plasma does not start to rise until 4-6 h after MI and measurement prior to this may give a false negative result. An elevated CK with a CK-MB fraction $>6 \%$ is highly suggestive of MI. However, a CK-MB fraction greater than $6 \%$ may also be seen following severe exercise, some surgical procedures, acute muscle injury or neuromuscular disease. In healthy children, the CK-MB fraction may be as high as $25 \%$, reducing its usefulness as an indicator of cardiac damage in this age group. ${ }^{20}$

\section{Quality control measures}

As described, there are potential problems with patient preparation, methodology and co-existing disease which can lead to pitfalls in the interpretation of the results of biochemical tests. But what care is taken to ensure that the results produced by the laboratory are accurate and precise? Laboratories run internal quality controls by including standards with known values amongst patients' samples at regular intervals. It is compulsory to participate in external quality assessment schemes, whereby samples containing unknown amounts of substance must be measured and the values obtained reported to external assessors. Laboratories failing to reach satisfactory standards have their practice reviewed.

\section{Conclusion}

Simple biochemical tests, if correctly performed and interpreted, are of help in the management of patients. Over-interpretation may lead to unnecessary further investigation but, to the astute, a biochemical abnormality may be the first manifestation of a previously unsuspected disease process.

8 Seah TG, Lew TG, Chin NM. A case of pseudohyperkalaemia and thrombocytosis. Acad Med Singapore 1998;27:442-3.

9 Naparstek Y, Gutman A. Case report: spurious hypokalaemia in myeloproliferative disorders. Am f Med Sci 1984;288:175-7.

10 Mayersohn M, Conrad KA, Achari R. The influence of a cooked meat meal on creatinine plasma concentration and creatinine clearance. Br f Pharmacol 1983;15:227-30.

11 Renoe BW, McDonald JM, Ladenson JH. The effect of stasis with and without exercise on free calcium, various cations and related parameters. Clin Chem Acta 1980;103:91-100.

12 Hawkins RC. Pseudohyperphosphataemia in multiple myeloma. Ann Clin Biochem 1991;28: 226-8

13 Burrin JM, Price CP. Measurement of blood glucose. Ann Clin Biochem 1985;22:327-42.

14 Weissman $M$, Klein B. Evaluation of glucose determination in untreated serum samples. Clin Chem.1958;4:420-2.

15 Chan AYW, Cockram CS, Swaminathan R. Effect of delay in separating plasma for glucose measurement upon the interpretation of oral glucose tolerance tests. Ann Clin Biochem 1990; 27:73-4.

16 Burnett JR, Crooke MJ, Delahunt JW, Feek $\mathrm{CM}$. Serum enzymes in hypothyroidism. NZ Med f 1994;14:355-6.

17 Moss DW, Henderson AR, Kachmar JF. Enzymes. In: Tietz NW, ed. Fundamentals of clinical chemistry. Philadelphia: WB Saunders Company, 1987; pp346-421.

18 Azizi F. Gamma-Glutamyl transpeptidase levels in thyroid disease. Arch Intern Med 1982;142: 79-81.

19 Sherwood RA, Lambert A, Newham DJ, Wassif WS, Peters TJ. The effect of eccentric exercise on serum creatine kinase activity in different thnic groups. Ann Clin Biochem 1996;33:3249 .

20 Turnbull DM, Bindoff LA. Muscle disease. In: Marshall WJ, Bangert SK, eds, Clinical biochemistry: metabolic and clinical aspects. Edinburgh: Churchill Livingstone, 1995; pp 543-55. 